\title{
Improved Approach for Obtaining Rotational Components of Seismic Motion
}

Hongnan Li, Liye Sun and Suyan Wang

Dept. of Civil Engineering, Shenyang Architectural and Civil Engineering Institute, Shenyang, P. R. China

\begin{abstract}
The rotational component of seismic strong-motion is attracting attention since it is becoming evident that it may contribute considerably to the overall response of structures to earthquake motions. This paper presents an improved method for calculating the time histories of torsional and rocking components of ground motion corresponding to a set of three recorded orthogonal translational components. The mathematical model is based on a detailed representation of soil impedance and contributions of body waves. The dependence of the angle of wave incidence on the frequency of wave is properly given in the calculation of rotational components with consideration of critical incident angles. Numerical results of the torsion and rocking obtained from a set of three recorded translational components are also presented.
\end{abstract}

\section{INTRODUCTION}

The current processing of earthquake records provide information only about the three translational (two horizontal and one vertical) components of the ground motion, primarily because these are the only components that can be directly measured. However, the translational components during a seismic event are always accompanied by rotational components because of the traveling wave effects. Several studies [1-4] have shown the importance of rotational components in the seismic analysis and design of structures. It was shown [5] that during an earthquake, even symmetric structures can be expected to undergo substantial torsional excitation. The seismic design codes also prescribe "accidental eccentricity" in the design force calculations to account for the unknown torsional inputs and unintended eccentricity in the design of a building. However, no such provisions are made to account for the rocking components that can have a significant impact on the design of tall and rigid structures. One of the reasons why this input is not explicitly taken into account in the seismic design codes is the lack of reliable information on torsional ground spectra.

Newmark [6] was perhaps the first researcher to establish a simple relationship between the torsional and translational components of a motion. It was based on the assumption of a constant velocity of wave propagation. Newmark's idea was pursued further by other investigators who proposed procedures to obtain the seismic torsional response spectra from the time history records of horizontal components. Hart et al [7] differentiated numerically two orthogonal translational records and obtained the associated free field rotational motion. A similar technique was implemented by Nathan and MacKenzie [8] to generate the record of the torsional ground motion from the two components of the EI Centro earthquake. When available, the acceleration time histories recorded by strong motion differential arrays can be used to generate rotational components by numerical differentiation of horizontal components. This technique was used by Niazi [9] to estimate the torsional and rocking induced by differential displacements on long rigid foundations and by Oliveira and Bolt [10] to estimate the rotational components from five earthquake recorded at the circular array in Taiwan, SMART-1.

The torsional ground motion response spectra have been developed by Tso and Hsu [11] and Rutenberg and Heidebrecht $[12,13]$. For the input motion considered by these investigators, these spectra had a flat peak in the period of 0.2 to $0.6 \mathrm{sec}$. These periods' ranges have special significance for some high frequency structures, such as medium height structures, stiff structures such as nuclear safety shell and structures with eccentric layouts likely to be affected by torsional base input. 
Although it was used by several authors, the assumption of constant plane wave velocity of propagation made for calculating the rotational components time histories from the translational components is hard to justify. This velocity depends upon the frequency of the wave motion and the angle of incidence. More rational procedures have been developed by References [14-18] where the requirement of a constant plane wave velocity of propagation was relaxed and the dispersion and transient arrival times of waves in an elastic half-space were considered. However, these methods still assume that the angle of incidence is fixed and known. The angle of incidence also depends upon the frequencies of the impinging harmonics of the ground motion. For correct calculation of the rotational components from the corresponding translational components, this dependence of the angle of incidence as well as the velocity of propagation of the wave on the frequency of the harmonics constituting the ground motion at a site must be considered.

In this paper, a method to include this dependence correctly is presented. The proposed procedure allows one to include the effect of the relative contributions of the P and SV waves to calculate time histories of the rotational component.

\section{ROTATIONAL COMPONENTS DUE TO BODY WAVES}

We will consider that the seismic ground motion is generated by plane harmonic waves arriving at the site. The direction of propagation of the waves is assumed to lie in the vertical $(x, z)$ plane. The passage of the wave front will induce particle displacements in the plane perpendicular to the direction of propagation and along this direction. The particle displacements in the plane perpendicular to the direction of propagation are decomposed into in-plane components of amplitude $A_{S V}$ due to the SV waves and out-of-plane components of amplitude $A_{S H}$ due to the SH waves. The amplitude of the displacements in the direction of propagation due to the $\mathrm{P}$ waves is $A_{P}$. The incidence and reflection of the body waves will originate two rotational components of the ground motion at the free surface: $\Phi_{g z}$ and $\Phi_{g y}$. The component $\Phi_{g z}$, referred to as the torsional component, is the rotation about the $\mathrm{z}$ axis and the component $\Phi_{g y}$, referred to as the rocking component, is the rotation about the y axis.

\section{P Wave Incidence}

When the waves encounter the free boundary, a reflection process with mode conversion occurs. The $P$ wave will reflect as another $P$ wave with an angle of reflection, $\sigma_{0}$ equal to the angle of incidence plus an SV wave with an angle of reflection $G_{1}$. These two waves will be identified with the subscripts PP and SP, respectively. For harmonic waves of frequency $\omega$, the potential functions are:

$$
\begin{aligned}
& \varphi_{p}=A_{p} \exp i \omega\left(\frac{\sin \theta_{0}}{\alpha} x-\frac{\cos \theta_{0}}{\alpha} z-t\right) \\
& \varphi_{p p}=A_{p p} \exp i \omega\left(\frac{\sin \theta_{0}}{\alpha} x+\frac{\cos \theta_{0}}{\alpha} z-t\right) \\
& \psi_{s p}=A_{s p} \exp i \omega\left(\frac{\sin \theta_{1}}{\beta} x+\frac{\cos \theta_{1}}{\beta} z-t\right)
\end{aligned}
$$

where $\alpha$ and $\beta$ are, respectively, the speeds of the $\mathrm{P}$ and $\mathrm{S}$ waves.

The particle displacements $u, w$ in the $x, z$ directions, respectively, are given by

$$
u=\frac{\partial\left(\varphi_{p}+\varphi_{p p}\right)}{\partial x}+\frac{\partial \psi_{s p}}{\partial z}, \quad w=\frac{\partial\left(\varphi_{p}+\varphi_{p p}\right)}{\partial z}-\frac{\partial \psi_{s p}}{\partial x}
$$


According to Eqs. (1) and (2), the particle displacements are therefore derived as follows

$$
u=i \omega\left(\frac{\sin \theta_{0}}{\alpha} \varphi_{P}+\frac{\sin \theta_{0}}{\alpha} \varphi_{P P}+\frac{\cos \theta_{1}}{\beta} \psi_{S P}\right), w=-i \omega\left(\frac{\cos \theta_{0}}{\alpha} \varphi_{P}-\frac{\cos \theta_{0}}{\alpha} \varphi_{P P}+\frac{\sin \theta_{1}}{\beta} \psi_{S P}\right)
$$

Imposing the free shear stress condition at the ground surface:

$$
\left.\tau_{x z}\right|_{z=0}=\left[\frac{\partial w}{\partial x}+\frac{\partial u}{\partial z}\right]_{z=0}=0
$$

from the elastic theory, the rocking component is

$$
\Phi_{g y}=\frac{1}{2}\left(\frac{\partial w}{\partial x}-\frac{\partial u}{\partial z}\right)
$$

From Eqs. (3) and (6), the rocking component can be written as:

$$
\Phi_{g y}=i \omega \frac{\sin \theta_{0}}{\alpha}\left[(-i \omega)\left(\frac{\cos \theta_{0}}{\alpha} \varphi_{P}-\frac{\cos \theta_{0}}{\alpha} \varphi_{P P}+\frac{\sin \theta_{1}}{\beta} \psi_{S P}\right)\right]=\Phi_{g y}=\frac{i \omega}{c_{x}} w
$$

in which $c_{x}=\frac{\alpha}{\sin \theta_{0}}$

\section{SV Wave Incidence}

The SV wave will reflect as another SV wave with an angle of reflection, $\sigma_{0}$ equal to the angle of incidence plus a SP wave with an angle of reflection $6_{2}$. These two waves will be identified with the subscripts PS and SS, respectively. Then the potential functions are

$$
\begin{aligned}
& \psi_{S V}=A_{S V} \exp i \omega\left(\frac{\sin \theta_{0}}{\beta} x-\frac{\cos \theta_{0}}{\beta} z-t\right) \\
& \varphi_{P S}=A_{P S} \exp i \omega\left(\frac{\sin \theta_{2}}{\alpha} x+\frac{\cos \theta_{2}}{\alpha} z-t\right) \\
& \psi_{S S}=A_{S S} \exp i \omega\left(\frac{\sin \theta_{0}}{\beta} x+\frac{\cos \theta_{0}}{\beta} z-t\right)
\end{aligned}
$$

the meaning of $u, w$ is as same as above all. According to

$$
u=\frac{\partial \varphi_{P S}}{\partial x}+\frac{\partial\left(\psi_{S V}+\psi_{S S}\right)}{\partial z}, \quad w=\frac{\partial \varphi_{P S}}{\partial z}-\frac{\partial\left(\psi_{S V}+\psi_{S S}\right)}{\partial x}
$$

using Eqs. (4) and (8), one obtains:

$$
\begin{aligned}
\Phi_{g y} & =\frac{\partial w}{\partial x}=\frac{\partial^{2} \varphi_{P S}}{\partial z \partial x}-\frac{\partial^{2}\left(\psi_{S V}+\psi_{S S}\right)}{\partial^{2} x} \\
& =i \omega \frac{\cos \theta_{2}}{\alpha} i \omega \frac{\sin \theta_{2}}{\alpha} \varphi_{P S}-\left[\left(i \omega \frac{\sin \theta_{0}}{\beta}\right)^{2} \psi_{S V}+\left(i \omega \frac{\sin \theta_{0}}{\beta}\right)^{2} \psi_{S S}\right]
\end{aligned}
$$


According to Snell's law, $\frac{\sin \theta_{0}}{\beta}=\frac{\sin \theta_{2}}{\alpha}$, one can also obtain:

in which $c_{x}=\frac{\beta}{\sin \theta_{0}}$.

$$
\Phi_{g y}=\frac{i \omega}{c_{x}} w
$$

\section{SH Wave Incidence}

In the case of the incident $\mathrm{SH}$ wave, there is no mode conversion and the wave reflects as itself with a reflection angle equal to the incidence angle $\sigma_{0}$. The potential functions of incident and reflected waves are

$$
V_{S H}=A_{S H} \exp i \omega\left(\frac{\sin \theta_{0}}{\beta} x-\frac{\cos \theta_{0}}{\beta} z-t\right), \quad V_{S H^{\prime}}=A_{S H^{\prime}} \exp i \omega\left(\frac{\sin \theta_{0}}{\beta} x+\frac{\cos \theta_{0}}{\beta} z-t\right)
$$

The displacement field $v$ caused by the incident waves and reflected waves in y direction is:

$$
v=2 V_{S H}=2 A_{S H} \exp i \omega\left(\frac{\sin \theta_{0}}{\beta} x-t\right)
$$

Since u doesn't depend on the out-of-plane coordinate, using (11) and (12) the torsion $\Phi_{g z}$ is obtained by

$$
\Phi_{g z}=\frac{1}{2}\left(-\frac{\partial u}{\partial y}+\frac{\partial v}{\partial x}\right)=\left.\frac{1}{2} \frac{\partial v}{\partial x}\right|_{z=0}=\frac{\partial V_{S H}}{\partial x}=i \omega \frac{\sin \theta_{0}}{\beta} \frac{v}{2}=\frac{i \omega}{2 c_{x}} v
$$

in which $c_{x}=\frac{\beta}{\sin \theta_{0}}$.

We will assume that the component $\mathrm{u}, \mathrm{v}$ and $\mathrm{w}$ of the ground motion at the free surface are available from measurements. Eqs. (6), (10) and (13) could be used to define the rotational component. However, this is not yet feasible with the state-of-the-art seismology. Therefore, in order to apply these equations to define $\Phi_{y, z}$, we need to know the incident angle $6_{0}$. How to calculate unknowns will be the subject of the following development.

\section{ANGLE OF INCIDENCE}

Let

$$
\operatorname{tg} \bar{e}=\frac{w}{u}
$$

The P and SV waves need to satisfy two boundary conditions: free shear stress condition and the free normal stress condition at the ground surface:

$$
\begin{gathered}
K^{2}\left(\frac{\partial w}{\partial z}+\frac{\partial u}{\partial x}\right)-\left.2 \frac{\partial u}{\partial x}\right|_{z=0}=0 \\
\frac{\partial w}{\partial x}+\left.\frac{\partial u}{\partial z}\right|_{z=0}=0
\end{gathered}
$$

in which $K=\frac{\alpha}{\beta}$. 


\section{Incident Angle of $\mathbf{P}$ Wave}

We assume that only a $P$ wave is propagating. Using Eqs. (2) and (15), one can obtaine

$$
K^{2}\left(\frac{\partial w}{\partial z}+\frac{\partial u}{\partial x}\right)-2 \frac{\partial u}{\partial x}=\frac{\omega^{2}}{\alpha^{2}}\left[-K^{2}\left(\varphi_{P}+\varphi_{P P}\right)+2 \sin ^{2} \theta_{0}\left(\varphi_{P}+\varphi_{P P}\right)+K^{2} \sin 2 \theta_{1} \psi_{S P}\right]=0
$$

According to Snell's law $\frac{\sin 6_{0}}{\alpha}=\frac{\sin \theta_{1}}{\beta}$, Eq. (17) can be written as:

$$
\cos 26_{1}\left(\varphi_{P}+\varphi_{P P}\right)-\sin 26_{1} \psi_{S P}=0
$$

Using Eq. (2) and (16), one can obtain:

$$
\frac{\partial w}{\partial x}+\frac{\partial u}{\partial z}=\frac{\omega^{2}}{\alpha^{2}}\left[\sin 2 \theta_{0}\left(\varphi_{P}-\varphi_{P P}\right)-K^{2} \cos 2 \theta_{1} \psi_{S P}\right]=0
$$

That is:

$$
\sin 2 \theta_{0}\left(\varphi_{P}-\varphi_{P P}\right)-K^{2} \cos 2 \theta_{1} \psi_{S P}=0
$$

Solving the systems of equations (18) and (20), we obtain:

$$
\left\{\begin{array}{l}
\varphi_{P P}=\frac{\Delta_{P P}}{\Delta_{P}} \varphi_{P} \\
\psi_{S P}=\frac{\Delta_{S P}}{\Delta_{P}} \varphi_{P}
\end{array}\right.
$$

where

$$
\begin{gathered}
\Delta_{P}=K^{2} \cos ^{2} 2 \theta_{1}+\sin 2 \theta_{0} \sin 2 \theta_{1} \\
\Delta_{P P}=-K^{2} \cos ^{2} 2 \theta_{1}+\sin 2 \theta_{0} \sin 2 \theta_{1} \\
\Delta_{S P}=2 \sin 2 \sigma_{0} \cos 2 \theta_{1}
\end{gathered}
$$

Eqs. (21), (22) and (3) allow one to express the two components of the ground motion $u$ and $w$ in terms of the displacement fields of the incoming $P$ wave:

$$
\begin{aligned}
& u=i \omega \frac{\varphi_{P}}{\Delta_{P}} \frac{2 K^{2}}{\alpha} \sin 2 \theta_{1} \cos \theta_{0} \\
& w=-i \omega \frac{\varphi_{P}}{\Delta_{P}} \frac{2 K^{2}}{\alpha} \cos 2 \theta_{1} \cos \theta_{0}
\end{aligned}
$$

Using Eqs. (23), (24) and (14) leads to

$$
\operatorname{tg} \bar{e}=\frac{w}{u}=-\frac{1}{\operatorname{tg} 2 \theta_{1}}
$$

Introducing the following notations

$$
\operatorname{tg} \bar{e}=G, x=\sin \sigma_{0}
$$

and based on Snell's law: $\sin \sigma_{0}=K \sin \sigma_{1}$, Eq. (25) can be written as a polynomial in $\mathrm{x}$

$$
x^{4}-K^{2} x^{2}+\frac{K^{4}}{4\left(G^{2}+1\right)}=0
$$

This expression allows one to calculate the incident angle $G_{0}$, which is considered with the dispersion. 


\section{Incident Angle of SV Wave}

Similarly, through some derivations, the relations between the incident angles and frequency are obtained as follows

$$
\begin{array}{lll}
G=\frac{2 x \sqrt{1-K^{2} x^{2}}}{K\left(1-2 x^{2}\right)} & \sigma_{0}<\sigma_{c} \\
G=-\frac{2 x \sqrt{K^{2} x^{2}-1}}{i K\left(1-2 x^{2}\right)} & \sigma_{0}>\sigma_{c}
\end{array}
$$

where

$$
\begin{aligned}
& G=\operatorname{tg} \bar{e}=\frac{w}{u} \\
& x=\sin \theta_{0} \\
& \theta_{c}=\arcsin \left(\frac{\beta}{\alpha}\right) \text {--the incident critical angle. }
\end{aligned}
$$

Using Eqs. (26), (27) and (28), we can obtain the incident angle of body waves, $\sigma_{0}$. Once the angle is found, the rotational component $\Phi_{g y}, \Phi_{g z}$ can then be obtained directly from Eqs. (6), (10) and (13).

\section{NUMERICAL RESULTS}

For illustration, we have used the three translation components of 1951 EI Centro earthquake to obtain the time histories for the corresponding rotational components. The time histories of the three translation components and their respective power spectra are shown in Figure 1. The predominant frequencies in the two horizontal components are in the range of 2 to 5 cycles per second, whereas in the vertical component the predominant frequency is about 10 cycles per second.

To obtain the rotational components from these records, these translation motion time histories were first resolved into their harmonics at 2048 discrete frequencies by using the fast Fourier transform. It is assumed that the recorded motions are primarily generated by shear waves. Thus for each harmonic component, Eq. (27) and (28) are used to calculate the frequency dependent angle of incidence. Knowing the angle of incidence, the rotational components at the frequency of the harmonic are obtained from Eq. (10) for the rocking component and Eq. (13) for the torsional component. This process is used to define the Fourier spectra of the two rotational components at all discrete frequencies. The inverse Fourier transform of these spectra provides the time histories.

The time histories for the rocking and torsional components, obtained by this process, are shown in Figure 2. The corresponding power spectra for these are also shown in the adjacent figure. The comparison of these rotational component spectra with the spectra of the translation component shows that the rotational spectra have more energy in the high frequency range of 7 to $20 \mathrm{cps}$.

\section{ACKNOWLEDGEMENTS}

The research work in this paper was supported by the National Natural Science Foundation of china under Grant No. 50025823. This support is gratefully acknowledged. 

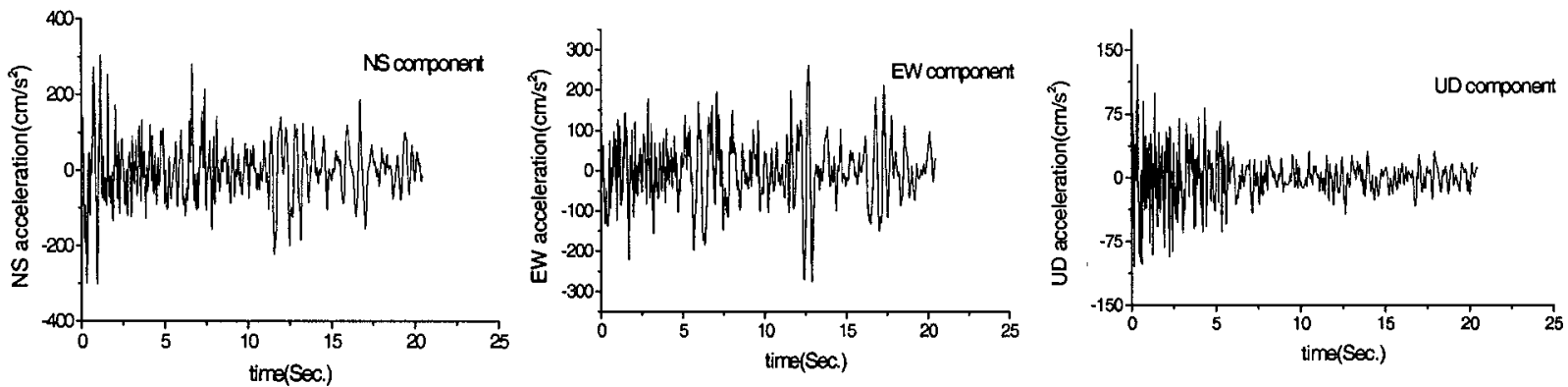

Fig. 1 (a) El Centro Translational Accelerations (Jan. 23, 1951)
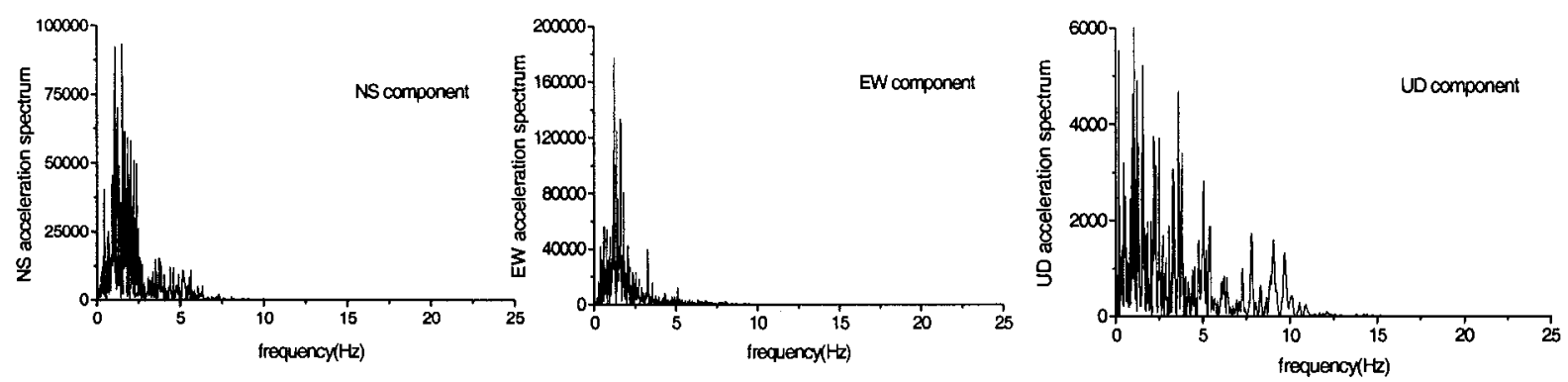

Fig. 1 (b) El Centro Translational Acceleration Power Spectrum (Jan. 23, 1951)

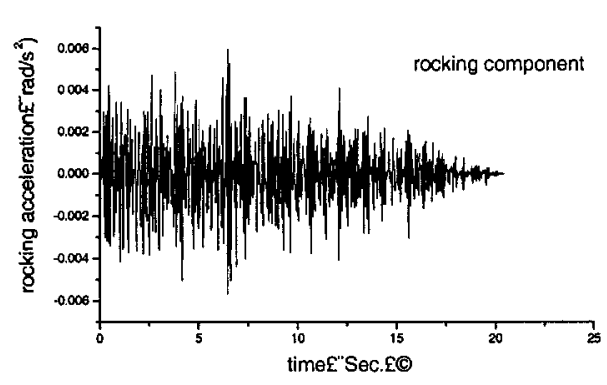

(a) Rocking Acceleration

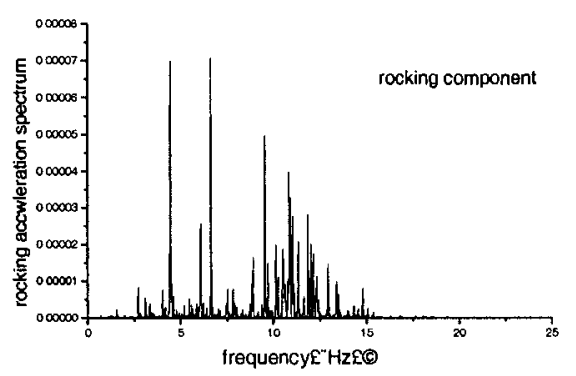

(c) Rocking Acceleration Power Spectrum

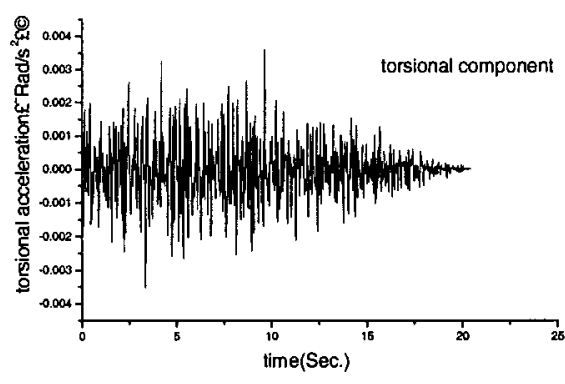

(b) Torsional Acceleration

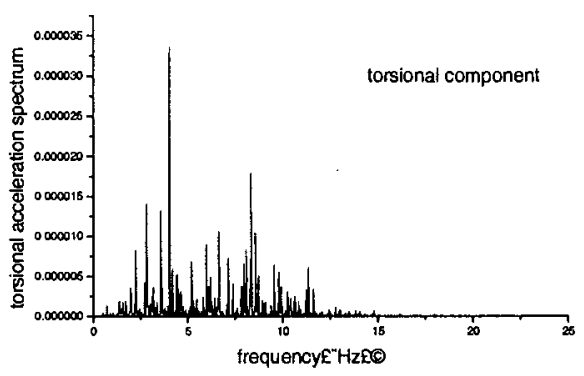

(d) Torsional Acceleration Power Spectrum

Fig. 2 El Centro Rotational Components (Jan. 23, 1951) 


\section{REFERENCES}

1. Bielak, J., "Dynamic Response of Nonlinear Building-Foundation System," Earthquake Engineering and Structural Dynamics, Vol. 6, 1978, pp. 17-30.

2. Abdel-Ghaffar, A. M. and Rubin, L. I., "Torsional Earthquake Response of Suspension Bridges," ASCE, Journal of Engineering Mechanics, Vol. 110, 1984, pp. 1467-1484.

3. Goel, R. K. and Chopra, A. K., "Dual-Level Approach for Seismic Design of Asymmetric-Plan Buildings," ASCE, Journal of Structural Engineering, Vol. 120, 1994, pp. 161-179.

4. Takeo, M., "Ground rotational motions recorded in near-source region of earthquakes," Geophysical Research Letters, Vol. 25, 1998, pp. 789-792.

5. Awad, A. M. and Humar, J. L, "Dynamic Response of Buildings to Ground Rotational Motion," Canadian Journal of Civil Engineering, Vol. 11, 1984, pp. 48-56.

6. Newmark, N. M., "Torsion in Symmetrical Buildings," Proc. of the $4^{\text {th }}$ World Conference on Earthquake Engineering, Vol. 2, pp. A3. 19-A3. 32, Santiago, Chile, 1969.

7. Hart, G. C., DiJulio, R. M., and Lew, M., "Torsional Response of High-Rise Buildings," ASCE, Journal of the Structural Division, Vol. 101, 1975, pp. 397-415.

8. Nathan, N. D. and MacKenzie, J. R., "Rotational Components of Earthquake Motions," Canadian Journal of Civil Engineering, Vol. 2, 1975, pp. 430-436.

9. Niazi, M., "Inferred Displacements, Velocities and Rotations of a Long Rigid Foundation Located at EI Centro Differential Array Site During the 1979 Imperial Valley, California Earthquake," Earthquake Engineering and Structural Dynamics, Vol. 14, 1986, pp. 531-542.

10. Olivera, C. S. and Bolt, B. A., "Rotational Components of Surface Strong Ground Motion," Earthquake Engineering and Structural Dynamics, Vol. 18, 1989, pp. 517-526.

11. Tso, W. K., and Hsu, T. -I., "Torsional Spectrum for Earthquake Motions," Earthquake Engineering and Structural Dynamics, Vol. 6, 1977, pp. 375-382.

12. Rutenberg, A. and Heidebrecht, A. C., "Rotational Ground Motion and Seismic Codes," Canadian Journal of Civil Engineering, Vol. 12, 1985, pp. 583-592.

13. Rutenberg, A. and Heidebrecht, A. C., "Response Spectra for Torsion, Rocking and Rigid," Earthquake Engineering and Structural Dynamics, Vol. 13, 1985, pp. 543-557.

14. Trifunac M. D., "A Note on Rotational Components of Earthquake Motions on Ground Surface for Incident Body Waves," Soil Dynamics and Earthquake Engineering, Vol. 1, 1982, pp. 11-19.

15. Lee, V. W. and Trifunac, M. D., "Torsional Accelerograms," Soil Dynamics and Earthquake Engineering, Vol. 4, 1985, pp. 132-139.

16. Lee, V. W. and Trifunac, M. D., "Rocking Strong Earthquake Accelerations," Soil Dynamics and Earthquake Engineering, Vol. 6, 1987, pp. 75-89.

17. Castellani, A. and Boffi, G., "Rotational Components of the Surface Ground Motion During an Earthquake," Earthquake Engineering and Structural Dynamics, Vol. 14, 1986, pp. 751-767.

18. Castellani, A. and Boffi, G, "On the Rotational Components of Seismic Motion," Earthquake Engineering and Structural Dynamics, 1989, Vol. 18, pp. 785-797. 